\title{
Common polymorphisms of the CFH, LOC 387715/ARMS2 and HTRA1 genes may not influence the intra-familial variability of $\mathrm{X}$-linked juvenile retinoschisis
}

\author{
BARKUR S. SHASTRY
}

Department of Biological Sciences, Oakland University, Rochester, MI, USA

Received February 2, 2010; Accepted February 26, 2010

DOI: $10.3892 / \mathrm{mmr} 00000282$

\begin{abstract}
X-linked juvenile retinoschisis (XLRS) is the leading cause of juvenile macular degeneration in males and is rare in females. Previous studies have shown that there is a marked intra- and inter-familial variation in disease severity and progression. This suggests that additional factors, such as genetic modifiers and environmental elements, influence disease severity. In order to understand the contribution of genetic modifiers, we aimed to ascertain whether common variants of the CFH, LOC 387715/ARMS2 and HTRA1 genes, which are major risk factors in age-related macular degeneration, contribute to the phenotypic variability of the XLRS disorder. Two unrelated XLRS families were selected, one harboring the missense mutation and the second a nonsense mutation in the RS gene. Both families exhibited variations in clinical phenotype. Genomic DNA from family members were analyzed for the above three genes using the polymerase chain reaction-based restriction fragment length polymorphism method. Our analyses revealed that both families were wild-type with respect to the LOC 387715/ARMS2 and HTRA1 genes. In one family (but not the other), the most severely affected and unaffected individuals were heterozygous for the $\mathrm{CFH}$ polymorphisms, while the less severely affected individual was wild-type. However, this alteration did not necessarily influence disease severity. Although we cannot completely rule out the role of the above genes in determining the phenotypic variability of the disorder, and though the statistical significance of the results could not be assessed due to the small scale of the study, it is unlikely that common polymorphisms of the CFH, LOC 387715/ARMS2 and HTRA1 genes serve as disease modifiers of the XLRS disorder.
\end{abstract}

Correspondence to: Dr Barkur S. Shastry, Department of Biological Sciences, Oakland University, Rochester, MI 48309, USA

E-mail: shastry@oakland.edu

Key words: degeneration, gene, juvenile, retinoschisis

\section{Introduction}

X-linked juvenile retinoschisis (XLRS), also known as congenital retinoschisis (RS), is a relatively rare recessively inherited bilateral vitreoretinal disorder that develops early in life. It is the leading cause of juvenile macular degeneration in males, and is rare in females. However, homozygous females and even, in rare cases, heterozygous females, may be affected (1). In the majority of cases, carriers have normal vision and cannot be identified by clinical means alone. The condition is characterized by the splitting of the superficial layer of the sensory retina in addition to macular degeneration (reviewed in ref. 2). Peripheral schisis may also occur in 50\% of cases (3). The disorder is clinically variable, and its prevalence ranges from 1:5,000 to $1: 25,000$. Visual impairment is usually mild in the early stages, but in the later stage vitreal hemorrhages, retinal detachment and occasionally neovascular glaucoma may develop, which may lead to blindness. The biochemical abnormality of RS is beginning to be understood.

Linkage mapping has allowed the identification and isolation of the gene underlying RS (termed RS1 or XLRS1), of which over 150 mutations have been reported $(4,5)$. The RS1 gene encodes a secreted protein and is expressed specifically in photoreceptors and within the inner portion of the retina (6). Retinoschisin, the $\mathrm{X}$-linked RS protein, contains an evolutionarily conserved discoidin domain, and diseasecausing mutations are clustered in this region (5). Biochemical studies on retinoschisin have demonstrated that the pathogenesis of RS is due to the misfolding of the discoidin domain, defective disulphide linked subunit assembly and the inability of retinoschisin to insert into the endoplasmic reticulum (7). Previous studies have shown that there is a marked intra- and inter-familial variation in disease severity. There is also a lack of correlation between mutation type and disease severity and progression (8-11). The intra-familial variation observed within the same germ-line mutation suggests that additional factors, such as genetic modifiers or environmental elements, influence disease severity. This has recently been further supported by a mouse model of RS (12). Although the phenotypes of RS (not a local degeneration in the macular area) and age-related macular degeneration (AMD) are quite different, and though there are few reasons to search for mutations in the AMD genes of RS patients, the possibility of contribu- 
Table I. Genotype of the RS patients at the CFH, LOC 387715/ARMS2 and HTRA1 loci.

\begin{tabular}{llllll}
\hline & & & & \multicolumn{2}{c}{ Genotype at locus } \\
\cline { 5 - 6 } Patients & Clinical phenotype & Mutation in the RS1 gene & CFH & LOC & HTRA1 \\
\hline Wild-type & - & - & $\mathrm{T} / \mathrm{T}$ & $\mathrm{G} / \mathrm{G}$ & $\mathrm{G} / \mathrm{G}$ \\
Family I & & & & \\
Patient 1 & Retinal detachment & R102Q & $\mathrm{T} / \mathrm{C}$ & $\mathrm{G} / \mathrm{G}$ & $\mathrm{G} / \mathrm{G}$ \\
Patient 2 & Foveal and peripheral schisis & R102Q & $\mathrm{T} / \mathrm{T}$ & $\mathrm{G} / \mathrm{G}$ & $\mathrm{G} / \mathrm{G}$ \\
Unaffected & Normal & None & $\mathrm{T} / \mathrm{C}$ & $\mathrm{G} / \mathrm{G}$ & $\mathrm{G} / \mathrm{G}$ \\
Family II & & & & & \\
Patient 1 & Retinal detachment & Y89X & $\mathrm{T} / \mathrm{T}$ & $\mathrm{G} / \mathrm{G}$ & $\mathrm{G} / \mathrm{G}$ \\
Patient 2 & Foveal schisis & Y89X & $\mathrm{T} / \mathrm{T}$ & $\mathrm{G} / \mathrm{G}$ & $\mathrm{G} / \mathrm{G}$ \\
\hline
\end{tabular}

\section{Family 1}

I

II

III

IV

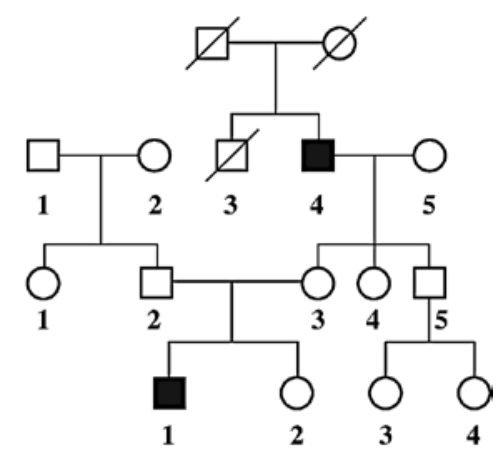

Family 2

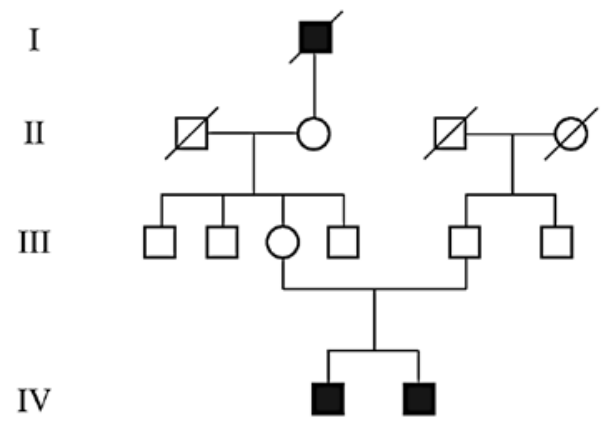

Figure 1. Pedigrees of RS families used in this study showing unaffected (open symbols) and affected (closed symbols) family members. Slashed symbols denote deceased individuals. In family 1 , the segregating RS mutation is R102Q; in family 2, it is Y89X.

tions of macular disease genes to phenotypic variability in RS cannot be ruled out. Therefore, we aimed to ascertain whether common variants of the CFH (Y402H), LOC 387715/ARMS2 (A69S) and HTRA1 (-512 G to A) genes, which are major risk factors in AMD (reviewed in ref. 13), contribute to the phenotypic variability of the XLRS disorder. As a prerequisite for more extensive studies to elucidate the role of genetic factors in the clinical variability of RS within the same genotype, the present small-scale study was carried out.
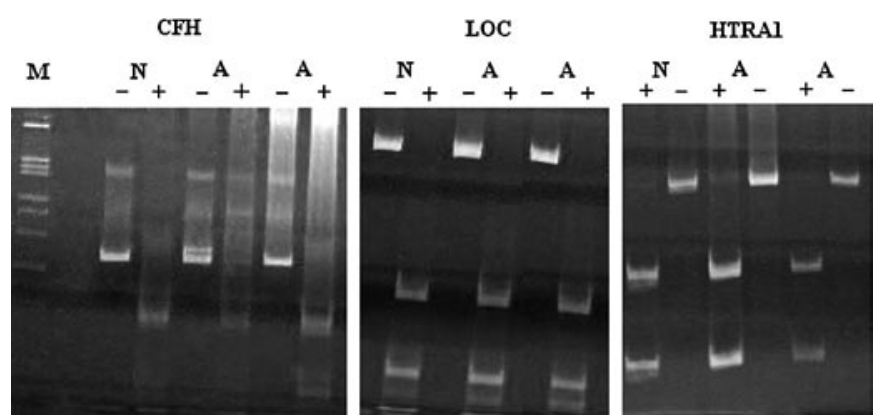

Figure 2. Restriction enzyme digestion pattern of PCR amplified products from normal and affected individuals (family 1). Only a portion of the analysis is shown. The amplified products in all three cases were cleaved by the respective enzymes. When the expected alterations in the three genes (Y402H in CFH, A69S in LOC 387715 and $-512 \mathrm{G}$ to A in HTRA1) are present, amplified DNA is not digested (they destroy the restriction sites in all three cases). N, normal; A, affected. (-), undigested DNA fragments; (+), digested DNA fragments.

\section{Patients and methods}

In order to validate our hypothesis and to further understand the genetic contribution to intra-familial phenotypic variability of the RS disorder, two unrelated families were selected (Fig. 1) harboring missense mutations (parent-offspring pair, family 1) and nonsense mutations (sib-sib pair, family 2) in the RS1 gene and exhibiting variations in clinical phenotype. Mutational analyses and the clinical phenotypes of these families were previously reported $(14,15)$, and are summarized in Table I. For genotype analysis, blood samples were collected, and leukocyte DNAs were isolated. Genomic DNA was amplified by the polymerase chain reaction (PCR) using three pairs of appropriate primers and conditions reported previously (16). The amplified product was subjected to restriction enzyme digestion as reported previously (16). The digestion products were analyzed on a $9 \%$ polyacrylamide gel and visualized by ethidium bromide staining. The study was approved by the Institutional Review Board of Oakland University, and all patients were informed of the aim of the study and gave their written consent for participation. 


\section{Results}

Variation observed within families having the same germ-line mutation indicates that other factors can influence disease expression in RS. In order to investigate the possible role of genetic modifiers, a small-scale study was carried out. Two families having a missense and a nonsense mutation (two extremes) were selected (Fig. 1). A portion of the restriction enzyme digestion pattern is shown in Fig. 2. The results revealed that all of the amplified samples (except in one patient) were cleaved by the respective enzymes, suggesting the absence of alterations in the restriction sites (mutations destroy the restriction site in each case). The genotype of the patients in each case is summarized in Table I. As can be seen in Table I, in family 1 a severely affected individual (maternal grandfather, Fig. 1) was heterozygous for the CFH variant $(\mathrm{Y} 402 \mathrm{H})$, whereas the less severely affected patient had a normal genotype (CFH panel, Fig. 2). In addition, the $\mathrm{CFH}$ mutation was also present in another unaffected individual (data not shown). This alteration was not noted in family 2 (sibling pair). Additionally, alterations in the LOC 387715/ ARMS2 (A69S) and HTRA1 (-512 G to A) genes were not found (Fig. 2) in any of the patients (in either of the families), and all patients had a normal genotype. These results suggest that common polymorphisms of these genes are not involved in determining intra-familial phenotypic variation in XLRS.

\section{Discussion}

In this small-scale study, we showed that common polymorphisms in the CFH, LOC 387715/ARMS2 and HTRA1 genes, which are major risk factors in AMD, may not contribute to intra-familial phenotypic variability in RS. The heterozygosity in the $\mathrm{CFH}$ gene in family 1 may not explain the severity of the disorder as i) the bilateral retinal detachment in this patient was recorded at the age of 40 and his severe condition could have been due to age-related problems, and ii) the unaffected individual of the same family also had this alteration. However, we cannot completely rule out the involvement of these genes, since mutations in other parts of the genes may contribute to the phenotypic variability or severity of disease. It is also difficult to confirm the involvement of a modifier gene in rare conditions. The statistical significance of this study was not determined due to the small-scale analysis. Further investigation and large-scale studies are required in order to understand the intra- and inter-familial variability of the RS disorder, and to validate the results of the present study. Identification of an RS modifier gene may provide a possible therapeutic target, as well as insight into the mechanisms of RS and another candidate susceptibility gene for XLRS.

\section{Acknowledgements}

I would like to thank Dr Michael T. Trese of William Beaumont Hospital, Royal Oak, MI for providing the blood samples from the patients and the clinical description of the disorder.

\section{References}

1. Mendoza KKT, Hiriyanna KT, Bingham BL, Rodriguez F, Shastry BS, Rodriguez A, Sieving PA and Tamayo ML: A Colombian family with X-linked juvenile retinoschisis with three affected females: finding of a frameshift mutation. Ophthalmic Genet 20: 37-43, 1999.

2. Shastry BS: Molecular genetics of X-linked juvenile retinoschisis. Curr Topics Genet 3: 37-43, 2008.

3. Sikkink SK, Biswas S, Parry NRA, Stanga PE and Trump D: X-linked retinoschisis: an update. J Med Genet 44: 225-232, 2007.

4. Sauer CG, Gehrig A, Warneke-Wittstock R, Marquardt A, Ewing CC, Gibson A, Lorenz B, Jurklies B and Weber BH: Positional cloning of the gene associated with $\mathrm{X}$-linked juvenile retinoschisis. Nat Genet 17: 164-170, 1997.

5. The Retinoschisis Consortium: Functional implications of the spectrum mutations found in 234 cases with X-linked juvenile retinoschisis (XLRS). Hum Mol Genet 7: 1185-1192, 1998.

6. Grayson C, Reid SNM, Ellis JA, Rutherford A, Sowden JC, Yates JRW, Farber DB and Trump D: Retinoschisin, the X-linked retinoschisis protein is a secreted photoreceptor protein and is expressed and released by Weri-Rb1 cells. Hum Mol Genet 9: 1873-1879, 2000.

7. Wu WW and Molday RS: Defective discoidin structure, subunit assembly and endoplasmic reticulum processing of retinoschisin are primary mechanisms responsible for X-linked retinoschisis. J Biol Chem 278: 28139-28146, 2003.

8. Eksandh LC, Ponjavic V and Ayyagari R, et al: Phenotypic expression of juvenile X-linked retinoschisis in Swedish families with different mutations in the XLRS1 gene. Arch Ophthalmol 118: 1098-1104, 2000.

9. Pimenides D, George ND, Yates JR, Bradshaw K, Roberts SA and Moore AT, et al: X-linked retinoschisis: clinical phenotypes and RS1 genotype in 86 UK patients. J Med Genet 42: e35, 2005.

10. Shinoda K, Ishida S, Oguchi Y and Mashima Y: Clinical characteristics of 14 Japanese patients with X-linked juvenile retinoschisis associated with XLRS1 mutation. Ophthalmic Genet 21: 171-180, 2000.

11. Nakamura M, Ito S, Terasaki H and Miyake Y: Japanese X-linked juvenile retinoschisis: conflict of phenotype and genotype with novel mutations in the XLRS1 gene. Arch Ophthalmol 119: 1553-1554, 2001.

12. Johnson BA, Aoyama N, Friedell NH, Ikeda S and Ikeda A: Genetic modification of the schisis phenotype in a mouse model of X-linked retinoschisis. Genetics 178: 1785-1794, 2008.

13. Shastry BS: Genetic risk factors in age-related macular degeneration. In: Retinal Degeneration: Causes, Diagnosis and Treatment. Catlin RB (ed). Nova Science publishers Inc, New York, pp108-118, 2009.

14. Hiraoka M, Rossi F, Trese MT and Shastry BS: X-linked juvenile retinoschisis: mutations at the retinoschisis and Norrie disease gene loci? J Hum Genet 46: 53-56, 2001.

15. Shastry BS, Hejtmancik FJ and Trese MT: Recurrent missense (R197C) and nonsense (Y89X) mutations in the XLRS1 genes in families with X-linked retinoschisis. Biochem Biophys Res Commun 256: 317-319, 1999.

16. Xu Y, Guan N, Xu J, et al: Association of CFH, LOC 387715 and HTRA1 polymorphisms with exudative age-related macular degeneration in a northern Chinese population. Mol Vis 14: 1373-1381, 2008. 